\title{
Elimination of porosity in bulk metallic glass castings using hot isostatic pressing
}

\author{
A.P. Srivastava ${ }^{a}$, M. Tong ${ }^{b}$, T. Ștefanov ${ }^{b}$ and D.J. Browne ${ }^{\star b}$ \\ aMaterials Science Division, Bhabha Atomic Research Centre, Trombay, Mumbai 400094, India

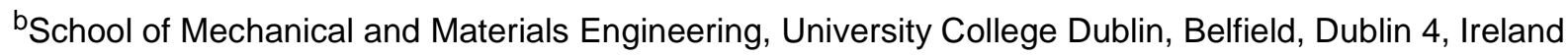 \\ *corresponding author: email: david.browne@ucd.ie
}

\begin{abstract}
:
This study presents design and implementation of a systematic method to remove the pores in as-cast bulk metallic glass using hot isostatic pressing, without changing the amorphous structure of the samples. The supercooled liquid region of $\mathrm{Zr}_{44} \mathrm{Cu}_{40} \mathrm{Al}_{8} \mathrm{Ag}_{8}$ was characterized using differential scanning calorimetry and dynamic mechanical analysis. This enabled informed choice of the range of hot isostatic pressing process variables likely to result in successful reduction of the porosity in the glassy alloy. The operating pressure in hot isostatic press processing was relatively less influential than either the temperature or the dwell time in controlling the porosity. It was shown that the dwell time should be longer than the average relaxation time in the glass transition range. With the specific bulk amorphous alloy under study, the optimized temperature, pressure and dwell time are $475^{\circ} \mathrm{C}, 50 \mathrm{MPa}$ and 3 minutes, respectively. Excess dwell times will result in crystallization.
\end{abstract}

Key words: Hot isostatic pressing; bulk amorphous alloys; porosity; design of experiments; supercooled liquid 


\section{Introduction:}

Recently, it has been reported that $\mathrm{Zr}$-Cu based bulk metallic glass (BMG) alloys possess large critical casting thickness, good strength, good corrosion resistance, low shrinkage upon cooling to room temperature and enhanced ductility at room temperature, making them potential contenders for advanced structural materials [1-4]. They have also exhibited high thermal stability as a supercooled liquid, above the glass transition temperature, and hence they offer better thermoplastic forming ability [4]. BMGs, unlike crystalline materials, have structural order that is limited to a few nanometers only and therefore sub-micrometer and nanoscale features can be made with precision on BMGs [5]. The possibility of plastic-like flow of a high strength material makes them suitable candidates for prospective industrial applications such as precision-shaped durable materials with complex surface features and patterns for micro-fluidic applications [4-9]. BMGs that are patterned with micro-features are being considered as inverted micro-structured tools for microinjection molding, which is one of the most popular mass production technologies for manufacturing relatively large thermoplastic polymer parts with small surface details and/or excellent surface finish, such as single-use micro-fluidic devices and micro-lenses [8, 9]. However, defect-free surfaces are needed for such high precision applications. If microporosity is present in as-cast BMG materials, the pores result in spherical voids at the surface of machined BMG prior to patterning [10]. A typical example of such a surface-breaking pore is shown in figure 1. It is known that micropores form during the process of rapid solidification [11, 12], yet that rapid solidification is the key process to successfully produce amorphous castings. The solution proposed here is to try to reduce, or preferably remove, the porosity formed during the casting of BMGs, using appropriate post-casting treatment. 


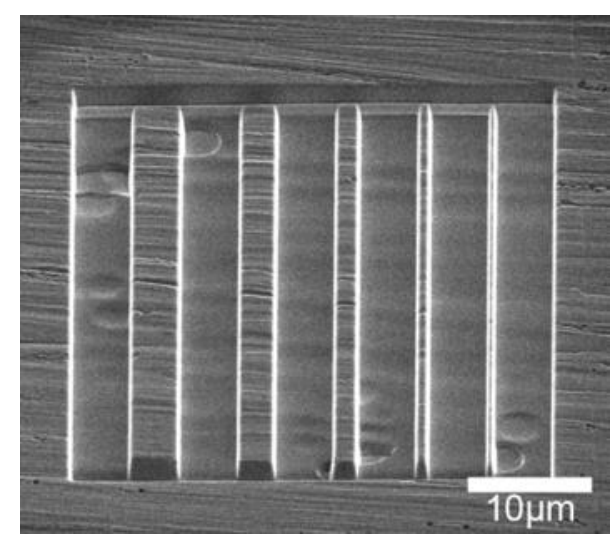

Fig 1: Surface-breaking pores on $\mathrm{Zr}_{47} \mathrm{Cu}_{45} \mathrm{~A} \mathrm{l}_{8} \mathrm{BMG}$ on which a series of channels and ridges of depth/height $\sim 2 \mu \mathrm{m}$ were machined using Focused lon Beam milling.

Hot Isostatic Pressing (HIPping) is a process that can be used to effectively close the pores of metallic materials - e.g. biomedical alloys [13] - and hence to achieve higher density. The key operation of HIPping involves pressuring the material with a high pressure inert gas at elevated temperature in a sealed enclosure. However, in the case of BMG, the processing window, in terms of time and temperature, is very restricted in order to avoid potential crystallization. This is due to the fact that BMG is relatively unstable at elevated temperature. To date, research reported on HIP treatment of BMGs is very limited. To the authors' knowledge, only Chen et al. [14] have presented the use of a HIPping technique to control the porosity of BMG. However, during their HIP processing, sample crystallization occurred and so the amorphous state of the BMG was destroyed.

In order to thermoplastically shape BMGs i.e. by processing in the regime in which the BMG behaves like a thermoplastic polymer, an understanding of the supercooled liquid region (SCLR) is of utmost importance. A metallic melt below the solidus temperature is a supercooled liquid and it can become metallic glass if it cools below a glass transition temperature $\left(T_{g}\right)$ without crystallization. This temperature is a dynamic temperature that depends on the cooling and heating rates during processing. 
A higher cooling or heating rate results in higher glass transition temperature. Near $T_{g}$, the supercooled liquid shows visco-elastic behavior, i.e. if the supercooled liquid is perturbed around $T_{g}$, the high frequency (short time) response can be elastic whereas the low frequency (long time) response can be viscous [4, 15-17]. Therefore, understanding of the supercooled liquid's relaxation time is very important when considering the stability of BMG. It has been observed that the relaxation time increases quite rapidly as the temperature is lowered $[15,17]$. The viscosity, which is a macroscopic measure of its resistance to flow, gives a good idea of relaxation time. The viscosity is found to be closely correlated to the relaxation time and they both increase as the temperature decreases [18]. This temperature dependence of the viscosity (and relaxation time) of supercooled liquids is often described by the Vogel-FulcherTammann (VFT) equation in metallic glasses $[16,17]$. Because metallic glasses have non directional metallic bonds, their viscosity rapidly increases with decreasing temperature, and hence they follow the VFT equation.

In this work, we report a systematic study of applying HIPping to the treatment of BMG alloys in an attempt to completely remove the pores that are formed during casting. We discuss the effect of HIPping process parameters viz. pressure, temperature and dwell time on reducing the porosity in BMGs. 


\section{Experimental Method}

An alloy of composition $\mathrm{Zr}_{44} \mathrm{Cu}_{40} \mathrm{Al}_{8} \mathrm{Ag}_{8}$ was prepared by melting pure constituent elements of purity $>99.99 \%$ in an arc melter in an argon gas atmosphere. The ingot was melted multiple times to ensure good compositional homogeneity of the alloy. This alloy was used to synthesize bulk metallic glass in the form of a rod, using a drop casting technique into a cooled copper mould in an argon gas atmosphere. The BMG rod was of $5 \mathrm{~mm}$ in diameter and $45 \mathrm{~mm}$ long. The amorphous nature of BMG was confirmed using X-ray diffraction; Siemens Kristalloflex with Cu-Ka radiation and Zeiss LIBRA 200 FE transmission electron microscope (TEM). Samples for TEM were prepared using Technoorg linda IV4 ion milling unit, at $4 \mathrm{KeV}$.

\subsection{Characterization of materials}

The viscosity of the BMG was measured at a variety of temperatures between $450^{\circ} \mathrm{C}$ and $470^{\circ} \mathrm{C}$ using dynamic mechanical analysis (DMA); Netzsch DMA 242 E Artemis. BMG samples were prepared with dimensions $55 \mathrm{~mm} \times 2 \mathrm{~mm} \times 3 \mathrm{~mm}$ machined from a separately cast BMG plate of the same composition. DMA was carried out using a 50 $\mathrm{mm}$ 3-point bending method in a nitrogen atmosphere.

Thermo-analytical studies were carried out in order to characterize the supercooled liquid (SCL) region of the BMG, using differential scanning calorimetry (DSC), Netzsch DSC 200f3. A continuous heating experiment at a constant rate of $10 \mathrm{~K} / \mathrm{min}$ was performed in the temperature range between $300^{\circ} \mathrm{C}$ and $525^{\circ} \mathrm{C}$ using the $\mathrm{DSC}$, in order to characterize the key temperatures of the BMG such as $T_{g}$ and the crystallization temperature $T_{x}$. For the purpose of testing the thermal stability of the BMG at elevated temperature, the BMG was also isothermally tested at temperatures of $460^{\circ} \mathrm{C}, 465^{\circ} \mathrm{C}$, $470^{\circ} \mathrm{C}$ and $475^{\circ} \mathrm{C}$ using the DSC.

\subsection{Processing as-cast BMG}

In order to conduct the HIP treatment and measure the porosity of the BMG, the $5 \mathrm{~mm}$ diameter rods were cut (through the cross-section) into discs of $3 \mathrm{~mm}$ thickness. These 
discs were subjected to mechanical grinding using grit sizes of P220, P320, P600, P1200, P2000, P2500, followed by diamond polishing via a conventional routine of preparing metallographic samples.

Following the characterization of the SCL region of the alloy, the temperature-time process window for HIPping of the amorphous BMG samples was determined. HIPping was carried out in an EPSI hot isostatic press at the Advanced Materials and Processing Laboratory of the University of Birmingham, UK, at various temperatures between $460^{\circ} \mathrm{C}$ to $480^{\circ} \mathrm{C}$, under pressures ranging from $50 \mathrm{MPa}$ to $180 \mathrm{MPa}$, and for different durations: ranging from 1 minute to 10 minutes, in an argon atmosphere. The details of the experiments are given in Table 1.

\begin{tabular}{|c|c|c|c|c|c|c|}
\hline $\begin{array}{c}\text { Experiment } \\
\text { Number }\end{array}$ & $\begin{array}{c}\text { Temperature } \\
\left({ }^{\circ} \mathrm{C}\right)\end{array}$ & $\begin{array}{c}\text { Applied } \\
\text { Pressure } \\
(\mathrm{MPa})\end{array}$ & $\begin{array}{c}\text { Dwell } \\
\text { time } \\
(\text { minutes })\end{array}$ & $\begin{array}{c}\text { Number } \\
\text { of } \\
\text { surfaces } \\
\text { examined }\end{array}$ & $\begin{array}{c}\text { Number } \\
\text { of pores } \\
\text { identified }\end{array}$ & $\mathrm{m}(\%)$ \\
\hline 1 & 460 & 50 & 10 & 20 & 121 & 84.5 \\
\hline 2 & 460 & 150 & 10 & 17 & 44 & 86.9 \\
\hline 3 & 465 & 50 & 5 & 15 & 32 & 87.2 \\
\hline 4 & 465 & 150 & 5 & 15 & 24 & 93.0 \\
\hline 5 & 472 & 180 & 3 & 15 & 0 & 100.0 \\
\hline 6 & 475 & 50 & 3 & 15 & 0 & 100.0 \\
\hline 7 & 475 & 180 & 1 & 19 & 123 & 80.0 \\
\hline 8 & 480 & 180 & 1 & 19 & 20 & 96.8 \\
\hline
\end{tabular}

Table 1:

HIPping experimental parameters, and their effect on porosity and the percentage change $(M)$ in pore area fraction.

Each HIPping experiment was performed in five stages, as follows.

- samples were heated to $430^{\circ} \mathrm{C}$ at a maximum possible heating rate of $20 \mathrm{~K} / \mathrm{min}$

- heating rate was then set to $10 \mathrm{~K} / \mathrm{min}$ until the temperature reached $450^{\circ} \mathrm{C}$ 
- heating rate was changed to $5 \mathrm{~K} /$ min until the target temperature was reached. The pressure of $10 \mathrm{MPa}$ was applied at the start of the second segment and the rate of increase of pressure was selected in such a way that the target pressure was achieved at the end of this third segment so that target temperature and target pressure were reached simultaneously

- samples were kept at constant pressure and constant temperature for the preselected dwell time

- finally, the pressurized chamber was depressurized and strong cool argon gas jets were applied to the BMG samples in order to quench them. The maximum cooling rate was measured to be around $7.2 \mathrm{~K} / \mathrm{min}$ in the process of quenching.

The evaluation of porosity in the BMG samples was performed using optical microscopy with the help of freeware Image J. To gather good statistics in the HIPped samples, each disc sample was also analyzed following serial sectioning in thickness steps of 20 $\mu \mathrm{m}$ and polishing. In this way, at least 4 surface layers were studied from each sample. There were at least 4 disc samples subjected to each HIP condition. For each set of HIPping experiments, a surface area of at least $2.94 \times 10^{8} \mu \mathrm{m}^{2}$ was thus analyzed, providing a good number of measurements to provide statistical certainty on the effects of HIPping on the BMG material, assuming pores are randomly distributed throughout the thickness of the rods.

In order to view the pores at higher resolution and to confirm they were indeed semicircular (indicative of gas porosity), a representative sub-set of them was selected for scanning electron microscopy (SEM); Hitachi TM-1000. These samples were cleaned with acetone using a lint-free cloth (ultra-soft and non-abrasive) and gold-sputtered prior to SEM. Also, to assess the depth and 3D profile of the pores, a typical one was selected for 3D optical profilometry; Veeco WYKO NT1100. 


\section{Results and discussions:}

\subsection{As-cast BMG}

The as-cast BMG sample was characterized using X-ray diffraction and the resultant diffractogram is shown in figure 2 . The absence of any crystalline peak confirms the amorphous nature of the as cast BMG.

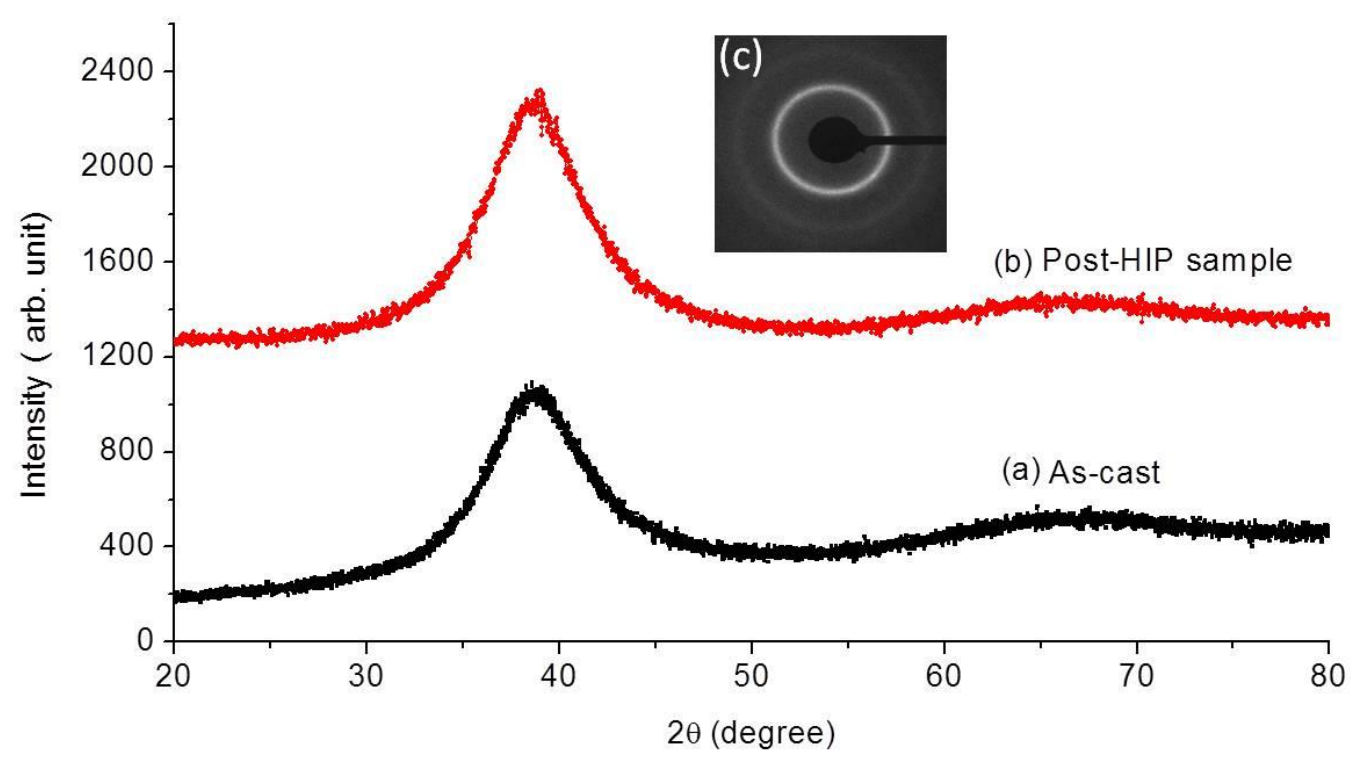

Fig. 2: X-ray diffractogram showing amorphous nature of (a) as-cast BMG and (b) postHIPping sample after experiment number 6 (Table 1); (c) Selected area electron diffraction pattern from HIPped sample.

The pores formed during the casting of the BMG sample were characterized using optical microscopy, following serial sectioning (in steps of $20 \mu \mathrm{m}$ ) and polishing. Figure 3 shows the normalized distribution of pores sizes in as-cast samples. It was observed that most of the pores were of size less than $20 \mu \mathrm{m}$. However, a small fraction of the 
pores of size $>50 \mu \mathrm{m}$ were also present. A typical micrograph of the surface of the polished BMG discs is shown in figure 4.

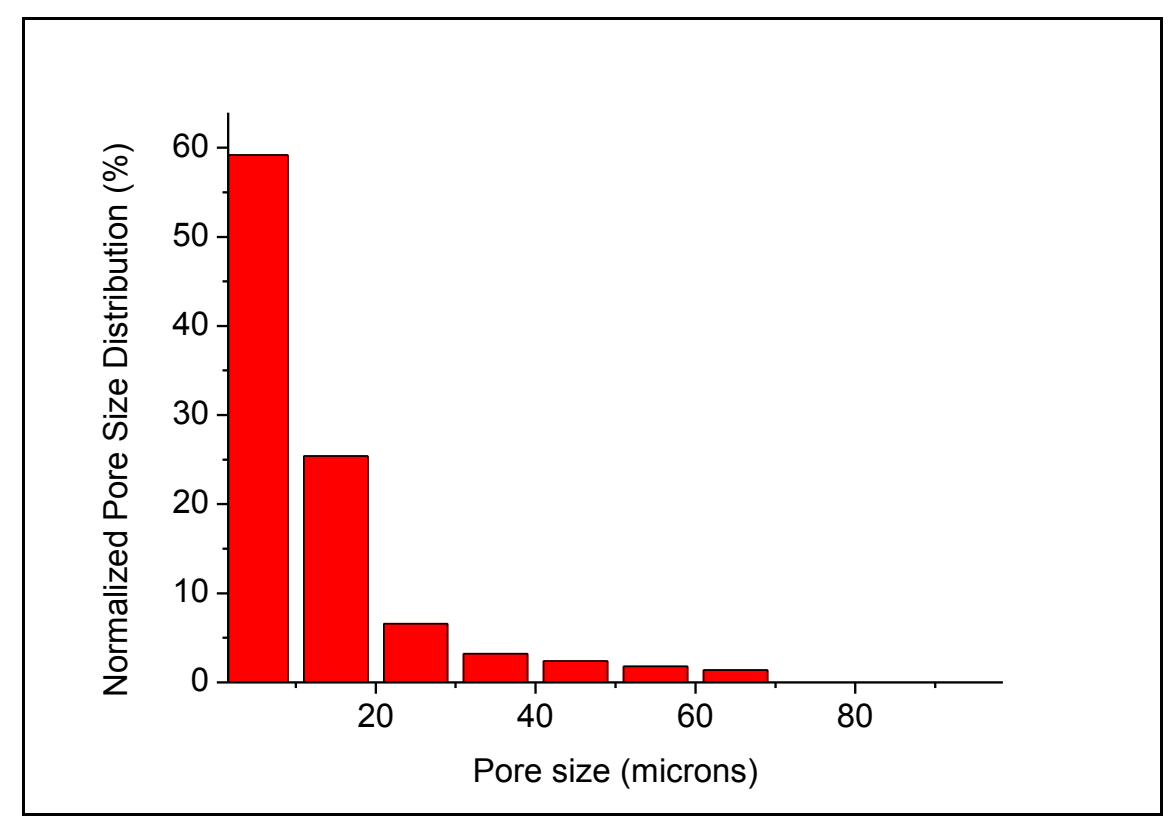

Fig.3: Normalized size distribution of pores in as-cast BMG. 


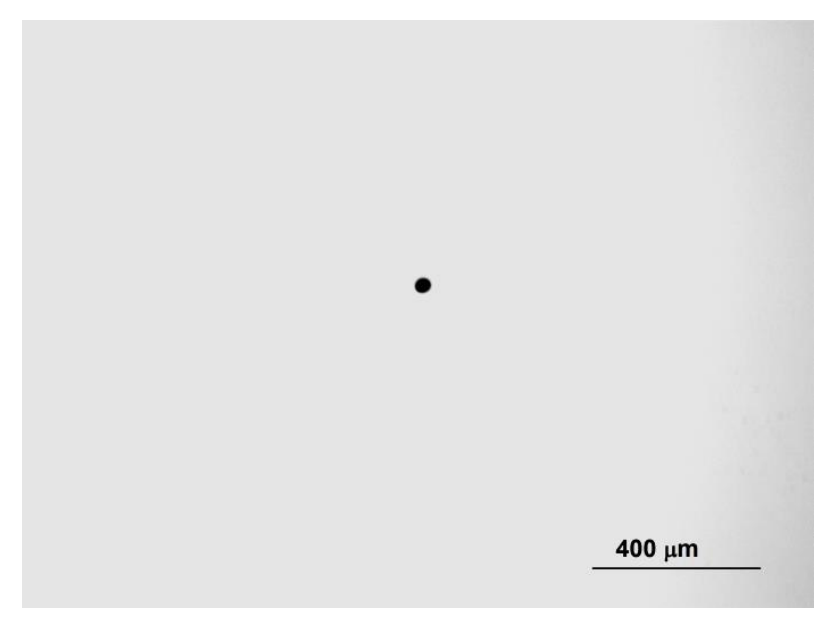

(a)

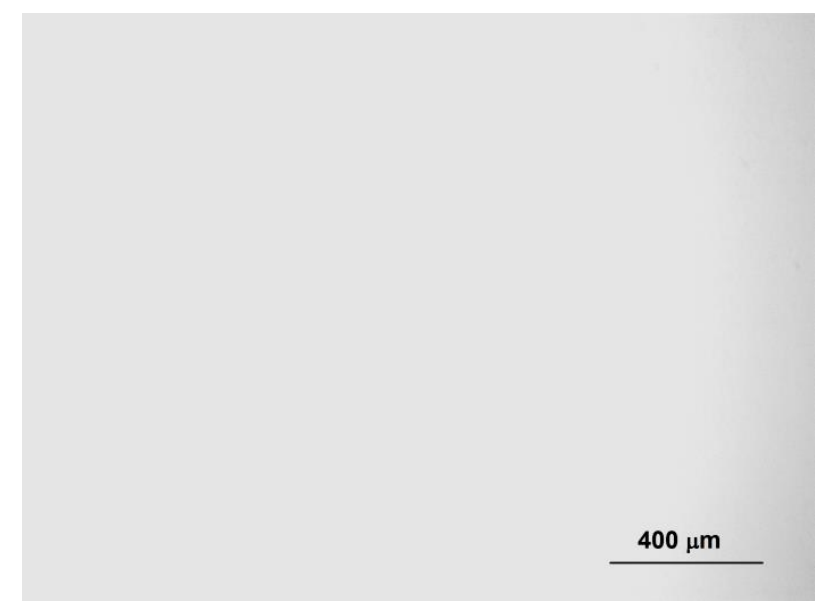

(b)

Fig.4: Typical optical micrograph obtained from (a) as cast sample (b) Post-HIP sample after experiment no. 6 described in Table 1.

Figure 5 shows the SEM images of two typical pores from HIPped specimens. In both cases they are about $10 \mu \mathrm{m}$ in diameter. They are clearly circular, indicative of a crosssection through a spherical pore. In one case (Fig 5a) the hole seems to be empty, but in the other (Fig $5 b$ ) the pore seems to be filled with material - probably abrasive particles from the grinding or polishing steps.

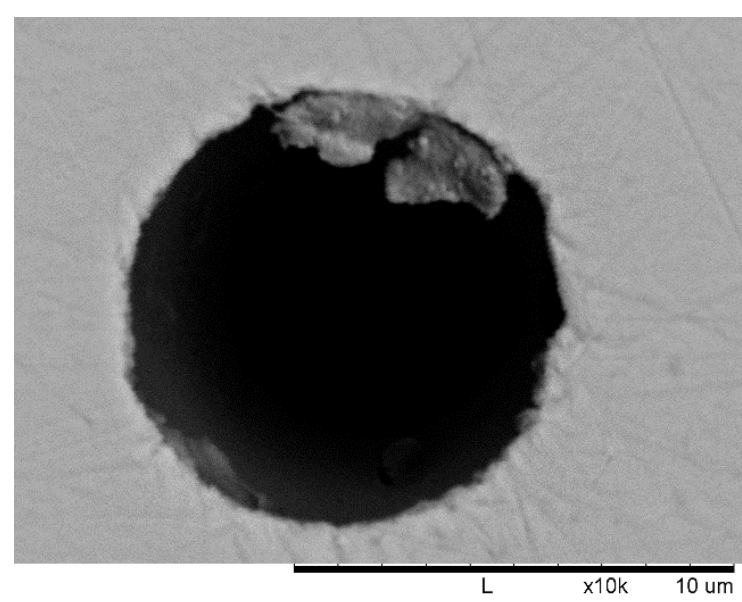

(a)

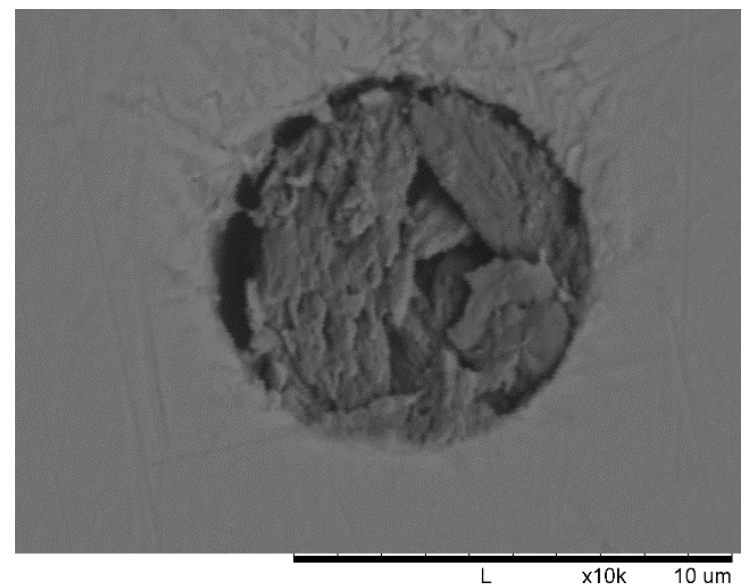

(b)

Figure 5. SEM images of (a) empty and (b) filled, pores in HIPped BMG samples. 
Changing the focus of the optical microscope also confirmed, visually, that identified pores indeed had depth.

Further confirmation that the surface features being counted (see Table 1) are indeed spherical pores, was provided by the results of 3D profilometry. Firstly, 2D line profiles were taken across the surface of the HIPped sample in a region that contains a pore: along the red (x profile) and blue (y profile) lines as shown in Figure 6 . Figures $7 \mathrm{a}$ and $7 \mathrm{~b}$ show the $x$ and $y$ profiles, respectively. The full profilometry scan produced the 3D profile shown in Fig. 8. It can be seen that this pore, of about $50 \mu \mathrm{m}$ diameter, has a depth of about $17 \mu \mathrm{m}$.

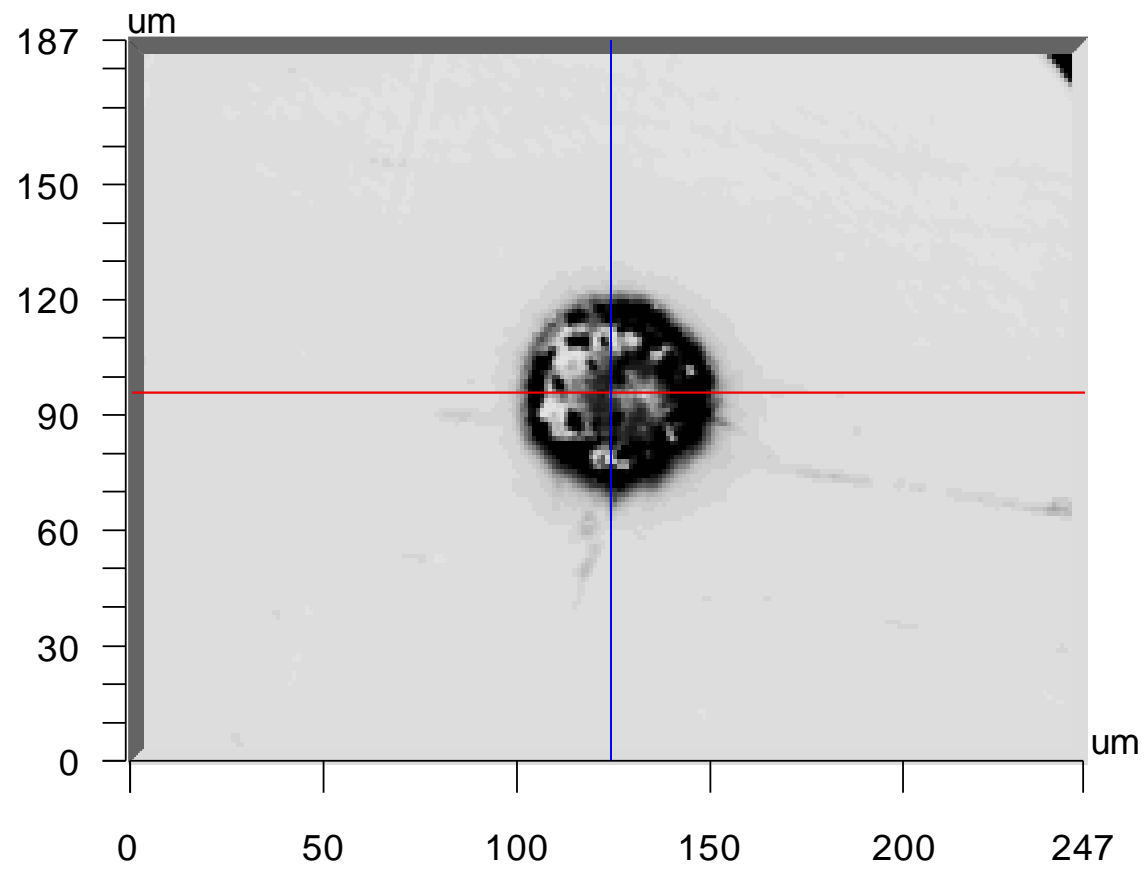

Figure 6 The red and blue lines indicate the $x$ and $y$ directions, respectively, along which line profiles were obtained for HIPped sample 

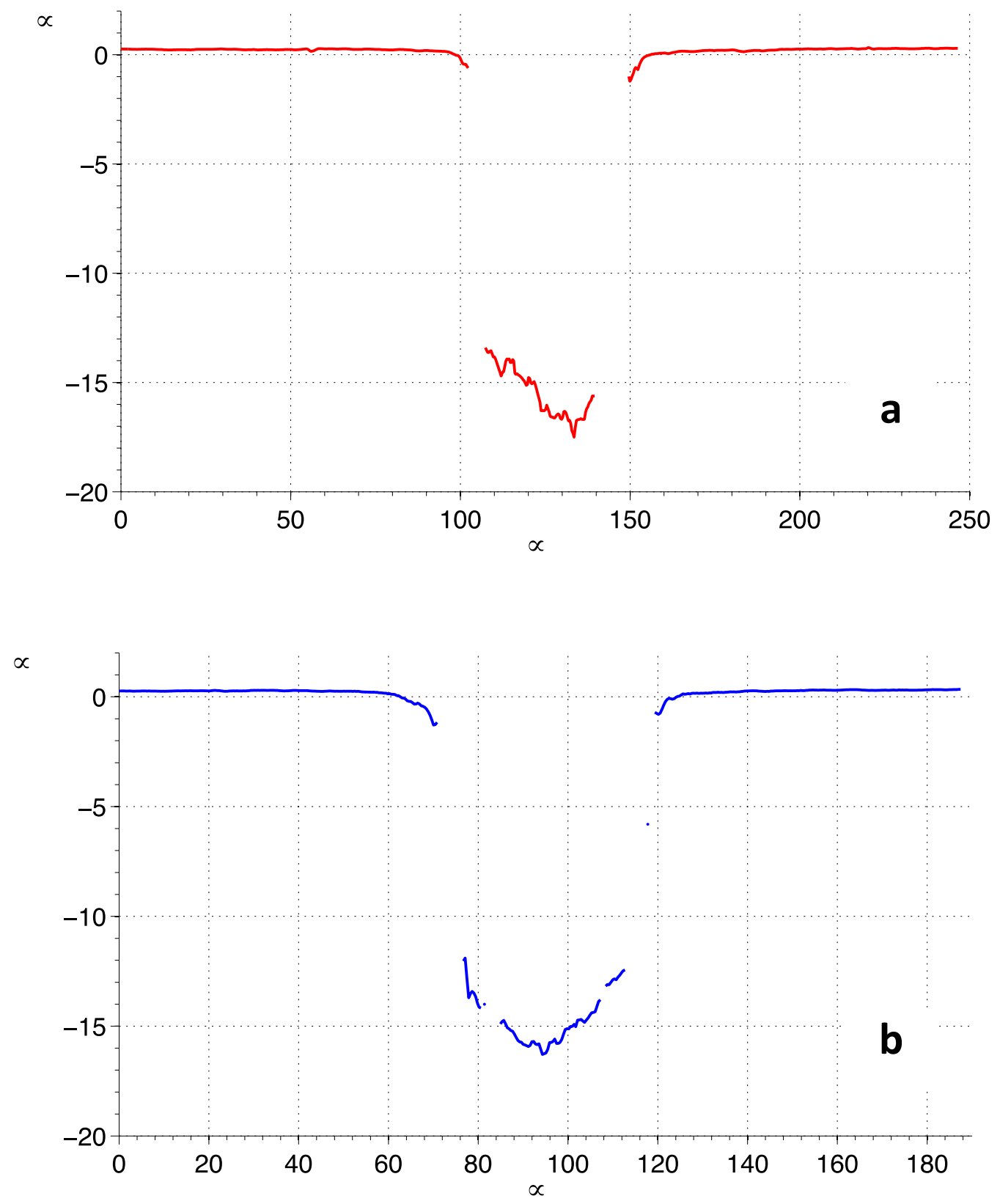

Figure $72 \mathrm{D}$ Optical profiles in the $\mathrm{x}(\mathrm{a})$ and $\mathrm{y}(\mathrm{b})$ directions along the red and blue lines indicated in Figure 6 


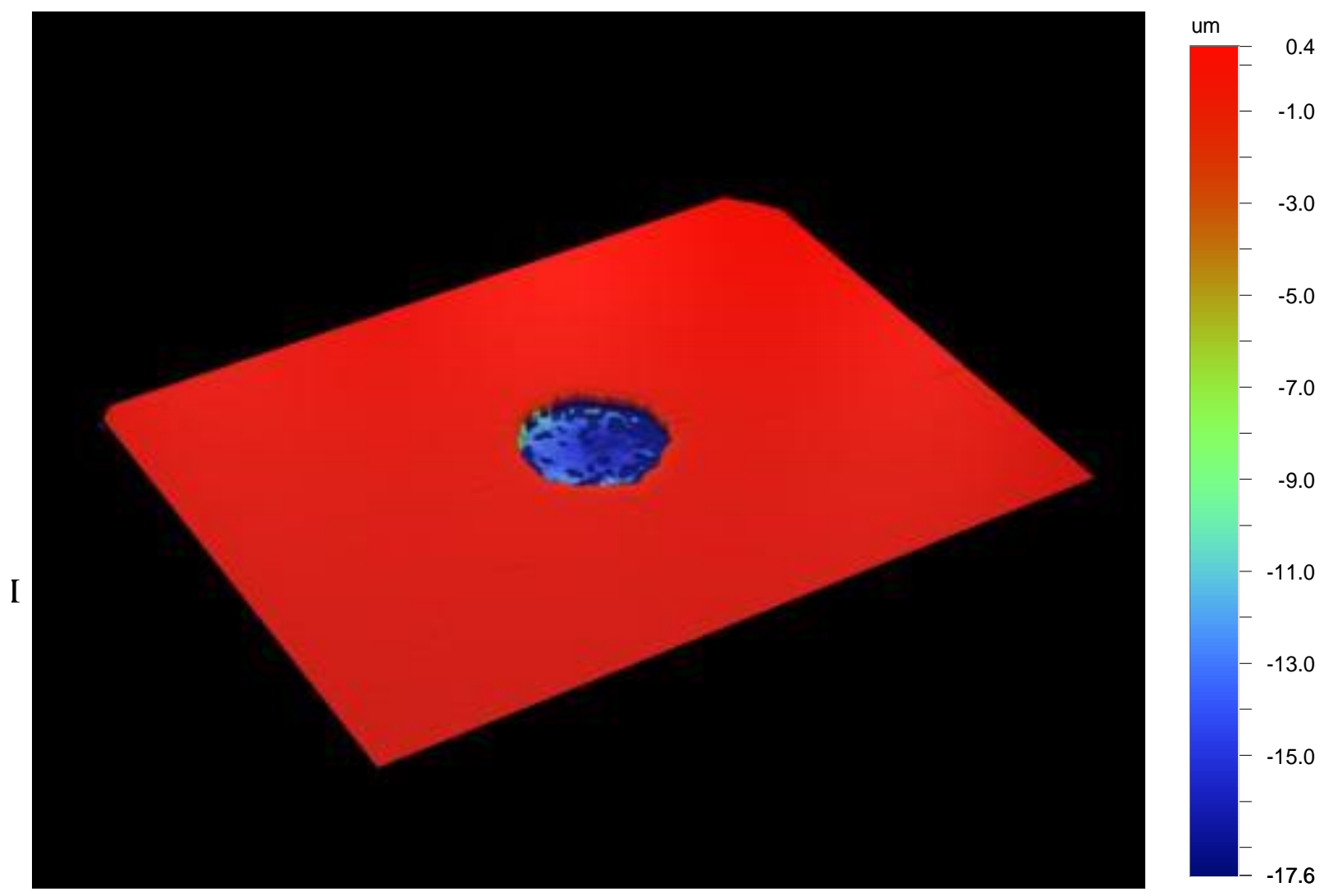

Figure 8: 3D surface profile of HIPed BMG sample, with pore in centre.

The thermal stability of the SCL region was studied using DSC. Figure 9 shows the DSC thermogram of the heat flow as a function of temperature when the sample was continuously heated at a constant rate of $10 \mathrm{~K} / \mathrm{min}$ between the temperatures $300^{\circ} \mathrm{C}$ and $525^{\circ} \mathrm{C}$. Inset in figure 9 shows a local zoom in of this thermogram between temperatures of $400^{\circ} \mathrm{C}$ and $490^{\circ} \mathrm{C}$. It can be seen, from this figure, that the glass transition onset and end temperatures are $426^{\circ} \mathrm{C}$ and $448^{\circ} \mathrm{C}$, respectively. The onset temperature and peak temperatures of crystallization process are $493^{\circ} \mathrm{C}$ and $501^{\circ} \mathrm{C}$, respectively. 


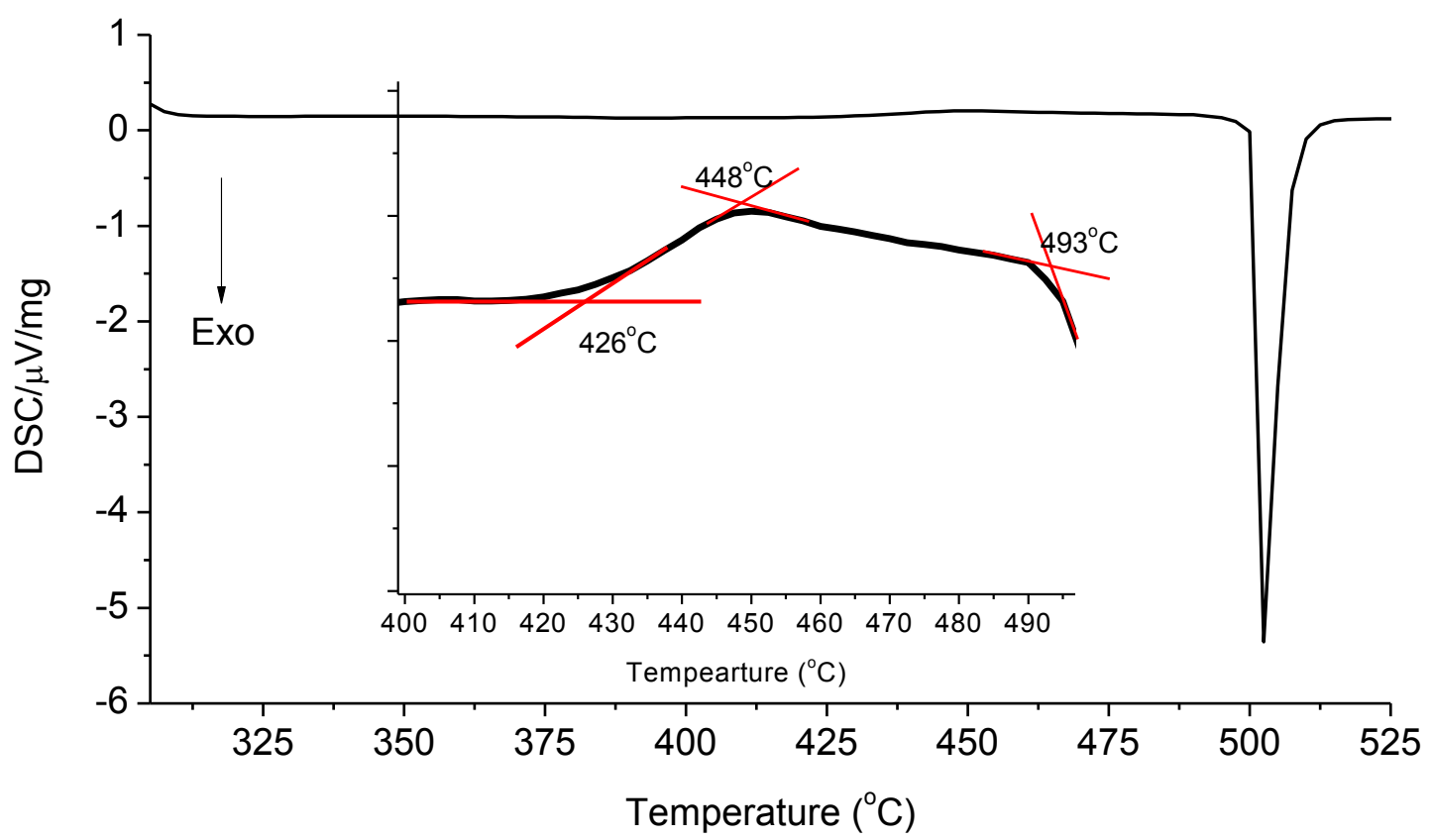

Fig. 9. DSC thermogram of as-cast BMG sample that was continuously heated at the constant heating rate of $10 \mathrm{~K} / \mathrm{min}$.

Therefore, the temperature range between $448^{\circ} \mathrm{C}$ and $493^{\circ} \mathrm{C}$ can be referred as the SCLR and the HIP treatment of the BMG has to be conducted in this range. It is known that viscosity of BMG is comparatively low at elevated temperature, facilitating the flow of the supercooled liquid, even with relatively low applied pressure. This makes the HIP treatment of the BMG more effective. However, at the elevated temperature, amorphous BMG becomes unstable and the crystallization becomes easier, therefore, reducing the processing time.

In order to characterize the thermal stability of the BMG, within the SCL region, isothermal experiments were performed at temperatures $460^{\circ} \mathrm{C}, 465^{\circ} \mathrm{C}, 470^{\circ} \mathrm{C}$ and $475^{\circ} \mathrm{C}$. The resultant DSC thermograms are presented in figure 10 . The onset time of the crystallization peaks at various temperatures is plotted in figure 11. It can be seen 
from this figure that at a temperature $460^{\circ} \mathrm{C}$ crystallization starts after $\sim 19$ minutes but this decreases to 4 minutes at $475^{\circ} \mathrm{C}$.

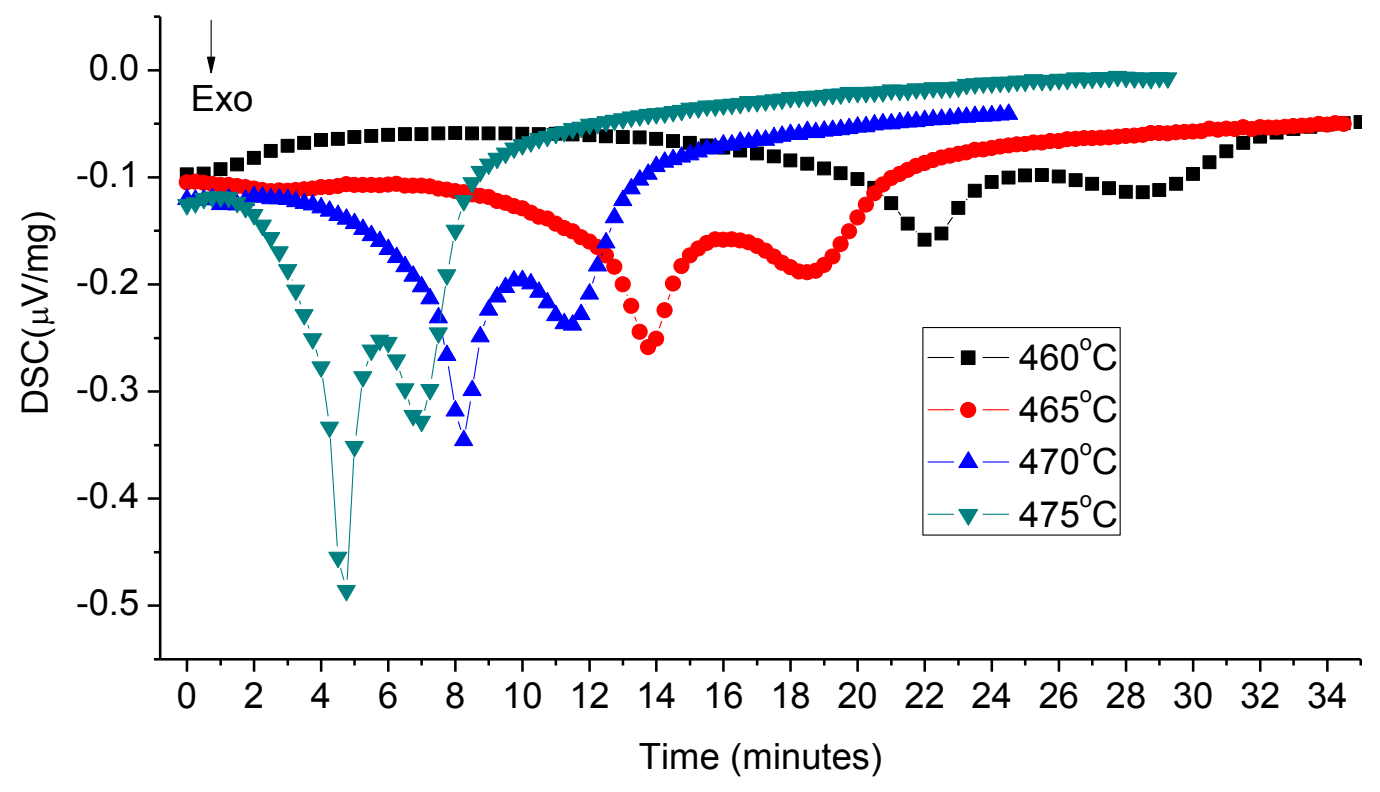

Fig. 10: DSC thermogram obtained from as-cast sample in isothermal runs taken at temperatures $460^{\circ} \mathrm{C}, 465^{\circ} \mathrm{C}, 470^{\circ} \mathrm{C}$ and $475^{\circ} \mathrm{C}$. 


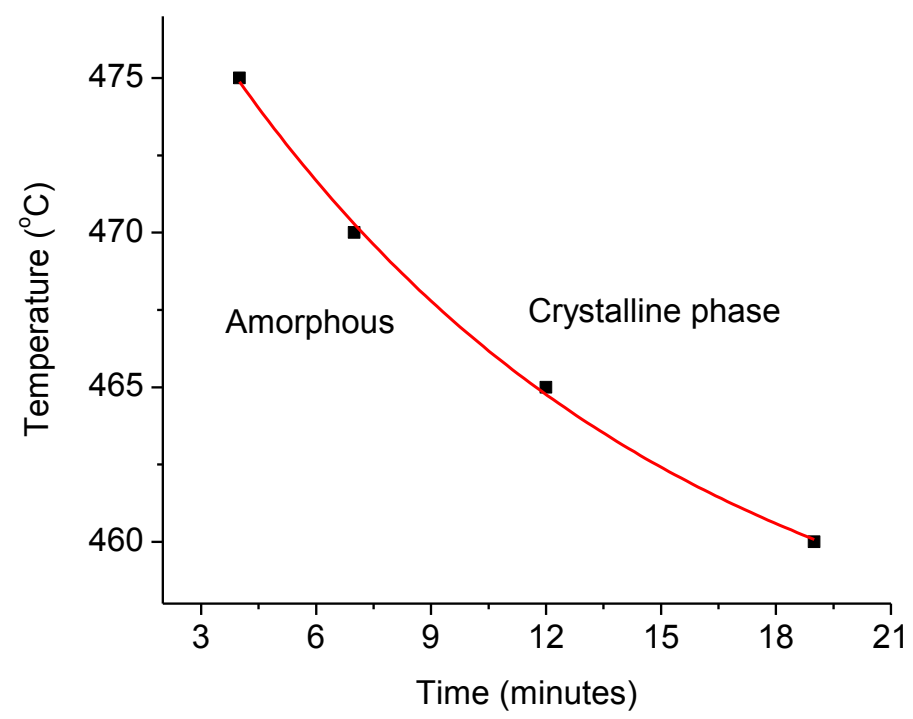

Fig. 11: The onset time of crystallization as a function of temperature obtained from isothermal run presented in fig. 6 .

The DMA study was used to characterize the viscosity of the BMG between temperatures of $450^{\circ} \mathrm{C}$ and $470^{\circ} \mathrm{C}$ as presented in fig. 12 . As stated, in metallic glasses, variation of viscosity with temperature in the SCLR is best described by VFT equation given in equation $1[19]$.

$$
\eta=\eta_{o} \cdot \exp \left(\frac{D^{*} \cdot T_{o}}{T-T_{o}}\right)
$$

where $D^{*}$ is known as fragility parameter and $T_{0}$ is the temperature at which viscosity approaches infinity; both are fitting parameters. The value of $\eta_{0}$ was set to 3.76 $\times 10^{-5} \mathrm{~Pa}$-s in accordance with the relation $\eta_{o}=N_{a} \cdot h / \mathrm{V}$, where $N_{a}$ is Avogadro's number, $h$ is the Planck constant and $V$ is the molar volume [20]. The solid curve in figure 12 shows the best fit of the VFT equation to experimental data available. The best fit gives $D^{*}=26$ and $T_{o}=323 \mathrm{~K}$. Similar values has been reported in literature for $\mathrm{Zr}$ based BMG $[20,21]$. It can be seen that at a temperature of about $470^{\circ} \mathrm{C}$, the viscosity was $\sim 4000$ 
MPa s. The time scale $\tau$ for viscosity relaxation can be estimated [16] by average relaxation time in the glass transition range, i.e.

$$
\tau=\frac{\Delta T}{\beta}
$$

where $\Delta T$ is the glass transition temperature region $\left(T_{g}^{\text {end }}-T_{g}{ }^{\text {onset }}\right)$ and $\beta$ is the heating rate, and therefore $\tau \cong 22 / 10=2.2$ minutes applies to the specific BMG under study.

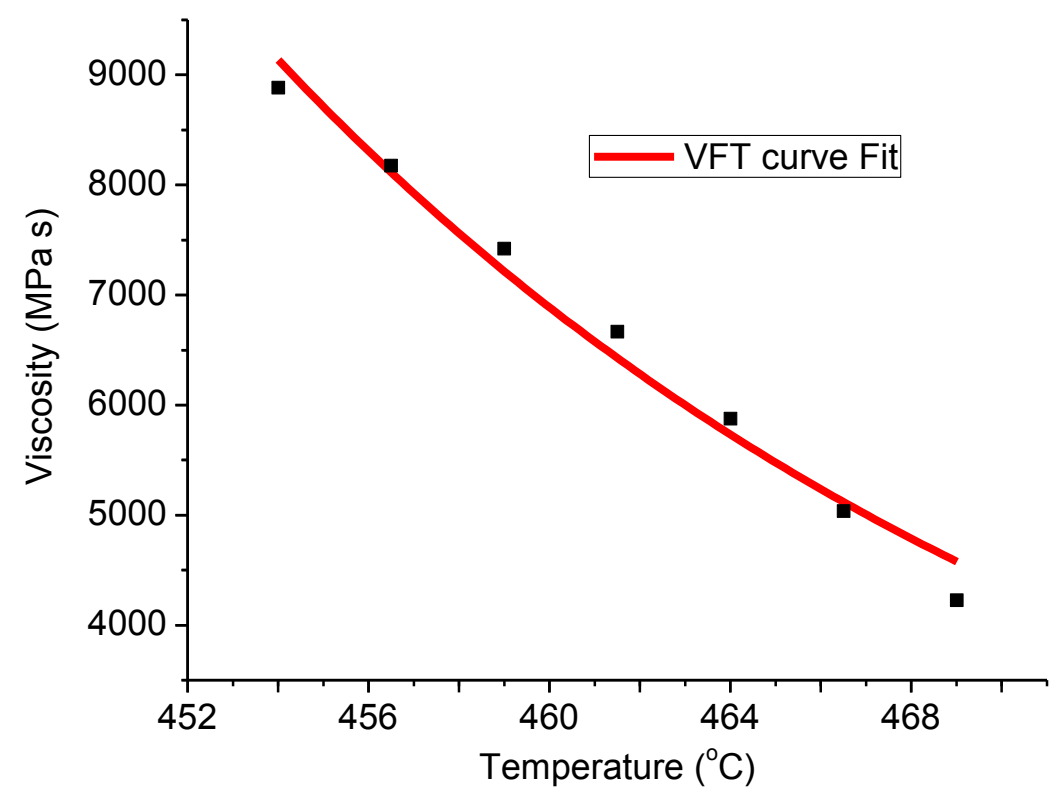

Fig. 12: Variation of viscosity as a function of temperature. Solid curve represents fitting of experimental data to VFT equation. 
3.2 BMG samples that were treated with HIP

In order to study the effect of HIPping experimental parameters, viz. temperature, pressure and dwell time, on porosity of BMG in the super cooled liquid state, the BMG samples were subjected to HIPping. The values for the experimental parameters were chosen following the characterization of the alloy's supercooled liquid. The temperatures for HIP testing were selected in SCLR namely $460^{\circ} \mathrm{C}, 465^{\circ} \mathrm{C}, 472^{\circ} \mathrm{C}$, $475^{\circ} \mathrm{C}$ and $480^{\circ} \mathrm{C}$. The dwell times at different temperatures were selected considering thermal stability of BMG at that temperature. Table 1 summarizes the experimental parameters for all the HIPping experiments. The samples after HIPping experiments were characterized using XRD, TEM, optical microscopy, SEM and optical profilometry, in order to confirm their amorphous state and measure the porosity.

Due to presence of residual air (albeit at very low levels) inside the chamber of the HIP device, the BMG samples were oxidized during the process of HIP. After the completion of HIP, the BMG samples were firstly polished and then tested with X-ray diffraction and TEM. A representative X-ray diffractogram and an electron microscope diffractogram of the BMG samples after HIP is shown in Fig.2b and $2 c$, respectively. It can be seen that there is no crystalline peak in the XRD pattern, which confirms that the BMG samples remained amorphous after the treatment with HIP. This is further confirmed by a diffuse halo in the selected area diffraction pattern obtained using TEM. XRD confirmed that all of the HIPped samples remained amorphous. The samples were then analyzed in an optical microscope for measurement of porosity. Quantification of porosity, representative of the bulk sample, was carried out via a method of serial sectioning wherein measurements were repeatedly obtained after removal, in sequential steps, of $20 \mu \mathrm{m}$ from the thickness of the BMG discs.

Table 1 gives the number of surfaces examined for each HIP experiment, and the total number of pores found for each HIP experiment. The average number of pores per surface examined therefore varies from $\sim 6(121 \div 20)$ for Experiment 1 , to 0 in Experiments 5 and 6 . This should be compared to the un-HIPped samples, where the average number of pores per surface examined was found to be $\sim 26$. The large number of surfaces examined per experimental condition means that they are 
statistically representative of the bulk sample, and that we are very unlikely to have missed unusual clusters of porosity.

A representative micrograph obtained from HIPping experiment number 6 (Table 1) is shown in figure $4(\mathrm{~b})$. In this paper, besides measurements of pore size, we calculated the average area fraction of pores $\left(F_{a v}\right)$ to characterize the porosity of the BMG. In order to quantify the change in porosity due to application of HIP, we define a parameter, which is the percentage reduction in average area fraction of pores after HIPping, ' $m$ ' as

$$
m=\frac{F_{a v(\text { ascast })}-F_{a v(H I P)}}{F_{a v(\text { ascast })}} \times 100 \%
$$

Figure 13 shows the result of the influence of the process parameter of HIP on the value of $m$.

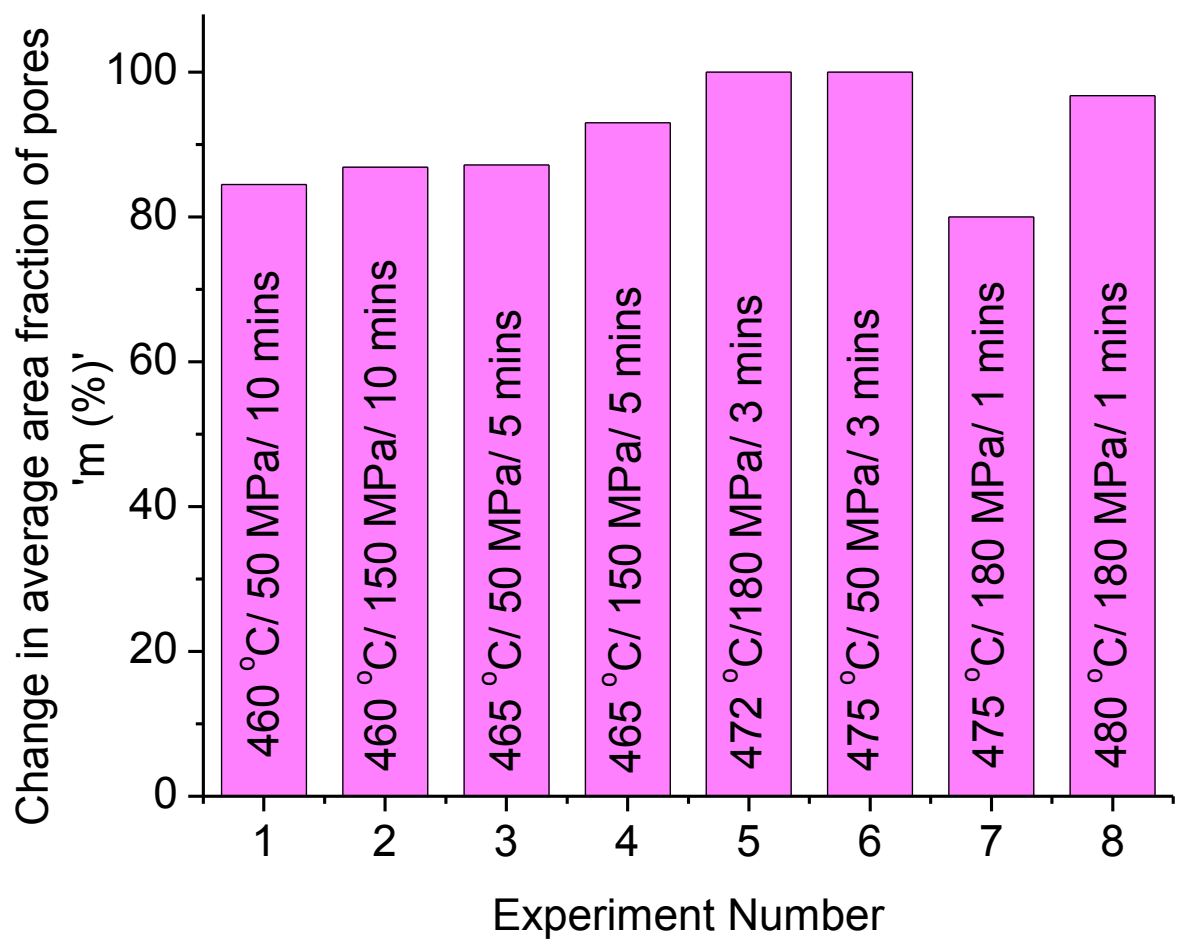

Fig. 13: The change of area fraction of pores after HIP at different operational parameters; experiments as per Table 1. 
It can be seen from fig. 13 that increase in pressure from $50 \mathrm{MPa}$ to 150 or $180 \mathrm{MPa}$ at a constant dwell temperature only resulted in a minor decrease of the porosity. On the other hand, it can be inferred from the same figure that, in general, the porosity decreases substantially with a small increase in the processing temperature. This is due to the fact that with the increase in the processing temperature, the viscosity of the BMG decreases rapidly (shown in Fig. 12; Eq.1), making the flow of supercooled liquid easier. However the result at the combination of $475^{\circ} \mathrm{C} / 180 \mathrm{MPa} / 1$ minute (experiment no. 7 ) deviates from this overall trend with a comparatively low porosity reduction of $80 \%$. The major difference between the operational parameters of this HIPping experiment and those of other experiments is the dwell time. In the run at $475^{\circ} \mathrm{C} / 50 \mathrm{MPa}$ (experiment no. 6 ), the dwell time is 3 minutes and $100 \%$ porosity can be removed even at lower pressure compared to experiment no. 7.This suggests the dwell time of one minute at $475^{\circ} \mathrm{C} / 180 \mathrm{MPa}$ is found to be too short and at the same temperature $475^{\circ} \mathrm{C} / 50 \mathrm{MPa}$, dwell time of three minutes was found to be sufficient to completely close all the porosity.

Although a significant decrease of the viscosity occurs with increasing temperature, sufficient dwell is essential for the viscosity of the supercooled liquid to decrease to a lower level and for the pores to shrink. An approximation for the minimum required dwell time can be made using average relaxation time in the glass transition range $\tau$ which is reported to be close to the time scale for viscosity relaxation. In the present case, $\tau \cong 2.2$ minutes (Eq.2), as previously calculated. Therefore, the HIP dwell time should be larger than 2.2 minutes, a dwell time of 1 minute as employed in experiment no. 7 is not long enough. However, excessive dwell times will lead to sample crystallization, as shown in Figs. 10 and 11. It is important to retain amorphicity in many resultant applications [6]. 


\section{Conclusions:}

Prior to the design of the HIP schedule, the supercooled liquid region of BMG $\mathrm{Zr}_{44} \mathrm{Cu}_{40} \mathrm{Al}_{8} \mathrm{Ag}_{8}$ alloy was characterized using DSC. The onset and end temperature of glass transition were found to be $426^{\circ} \mathrm{C}$ and $448^{\circ} \mathrm{C}$, respectively, at a heating rate of 10 $\mathrm{K} / \mathrm{min}$. The supercooled liquid region could be described as the temperature range between $448^{\circ} \mathrm{C}$ and $493^{\circ} \mathrm{C}$. The average relaxation time in the glass transition range was found to be 2.2 minutes. The HIPping study showed that the processing temperature and dwell time are the most influential parameters in controlling the porosity of the BMG. The dwell time can generally be selected larger than the average relaxation time in the glass transition range. The processing temperature should be selected inside the supercooled liquid region. Higher processing temperature is preferable to the flow of the supercooled liquid; however the processing time at that temperature should be optimized in order to avoid crystallization. We have shown that it is feasible to completely remove the porosity of $\mathrm{Zr}_{44} \mathrm{Cu}_{40} \mathrm{Al}_{8} \mathrm{Ag} 8 \mathrm{BMG}$ cast. With respect to this specific BMG, the optimized temperature, pressure and dwell time are $475^{\circ} \mathrm{C}, 50$ $\mathrm{MPa}$ and 3 minutes respectively. This defect-free material can now be used for precision engineering applications, which requires very low surface roughness and high durability.

Acknowledgements:

The authors would like to thank Enterprise Ireland for provision of funding to this project, under contract number CFTD/2012/2022. 


\section{References:}

[1] S. Cheng, C. Wang, M. Ma, D. Shan, B. Guo, Mechanism for microstructural evolution induced by high temperature deformation in Zr-based bulk metallic glasses, Journal of Alloys and Compounds, 676 (2016) 299-304.

[2] J.J. Fan, Y.F. Yan, S.H. Chen, C.-H. Ng, F.F. Wu, K.C. Chan, Reliability of the plastic deformation behavior of a Zr-based bulk metallic glass, Intermetallics, 74 (2016) 25-30.

[3] N.W. Khun, H. Yu, Z.Z. Chong, P. Tian, Y. Tian, S.B. Tor, E. Liu, Mechanical and tribological properties of Zr-based bulk metallic glass for sports applications, Materials \& Design, 92 (2016) 667-673.

[4] A.P. Srivastava, T. Ștefanov, D. Srivastava, D.J. Browne, Multiple relaxation processes in Zr44Cu40Al8Ag8 bulk metallic glass, Materials Science and Engineering: A, 651 (2016) 69-74.

[5] D.J. Browne, D. Stratton, M.D. Gilchrist, C.J. Byrne, Bulk metallic glass multiscale tooling for molding of polymers with micro to nano features: A review, Metallurgical and Materials Transactions A: Physical Metallurgy and Materials Science, 44 (2013) 2021 2030.

[6] N. Zhang, A. Srivastava, B. Kirwan, R. Byrne, F. Fang, D.J. Browne, M.D. Gilchrist, Manufacturing microstructured tool inserts for the production of polymeric microfluidic devices, Journal of Micromechanics and Microengineering, 25 (2015) 095005.

[7] J. Schroers, T.M. Hodges, G. Kumar, H. Raman, A.J. Barnes, Q. Pham, T.A. Waniuk, Thermoplastic blow molding of metals, Materials Today, 14 (2011) 14-19.

[8] N. Zhang, C.J. Byrne, D.J. Browne, M.D. Gilchrist, Towards nano-injection molding, Materials Today, 15 (2012) 216-221.

[9] N. Zhang, A.P. Srivastava, D.J. Browne, M.D. Gilchrist, Performance of nickel and bulk metallic glass as tool inserts for the microinjection molding of polymeric microfluidic devices, Journal of Materials Processing Technology, 231 (2016) 288-300.

[10] P.J. Meagher, D.J. Browne, Castability of bulk metallic glass materials for multiscale tooling applications, Proc. 8th Pacific Rim International Congress on Advanced Materials and Processing, 4-9 August 2013, Waikoloa, Hawaii, USA (Ed. F. Marquis), TMS, (2013) 3213-3220.

[11] Y.S. Chang, K.T. Hsu, J.B. Li, P.H. Tsai, J.S.C. Jang, J.C. Huang, Effect of cast process and microalloying on the fracture toughness of Zr-based bulk amorphous alloys, Journal of Alloys and Compounds, 614 (2014) 87-93.

[12] Y. Yokoyama, K. Fukaura, A. Inoue, Cast structure and mechanical properties of $\mathrm{Zr-Cu-Ni-Al} \mathrm{bulk} \mathrm{glassy} \mathrm{alloys,} \mathrm{Intermetallics,} 10$ (2002) 1113-1124.

[13] R. Kaiser, K. Williamson, C. O'Brien, S. Ramirez-Garcia, D.J. Browne, Effects of hot isostatic pressing and heat treatment on cast cobalt alloy, Materials Science and Technology, 31 (2015) 1298-1304.

[14] Q. Chen, D. Zhang, J.U.N. Shen, H. Fan, J. Sun, Z. Liu, Effect Of Hot Isostatic Pressing on mechanical properties of Fe41Cr15Mo14C15B6Y2Co7 BMG, International Journal of Modern Physics B, 20 (2006) 3617-3622.

[15] C.A. Angell, K.L. Ngai, G.B. McKenna, P.F. McMillan, S.W. Martin, Relaxation in glassforming liquids and amorphous solids, Journal of Applied Physics, 88 (2000) 31133157. 
[16] E. Pineda, P. Bruna, B. Ruta, M. Gonzalez-Silveira, D. Crespo, Relaxation of rapidly quenched metallic glasses: Effect of the relaxation state on the slow low temperature dynamics, Acta Materialia, 61 (2013) 3002-3011.

[17] J.C. Qiao, J.M. Pelletier, Dynamic Mechanical Relaxation in Bulk Metallic Glasses: A Review, Journal of Materials Science \& Technology, 30 (2014) 523-545.

[18] S. Webb, R. Knoche, $5^{\text {th }}$ Silicate Melt Workshop. The glass-transition, structural relaxation and shear viscosity of silicate melts, Chemical Geology, 128 (1996) 165-183.

[19] D.N. Perera, Compilation of the fragility parameters for several glass-forming metallic alloys, Journal of Physics: Condensed Matter, 11 (1999) 3807.

[20] R. Busch, E. Bakke, W.L. Johnson, Viscosity of the supercooled liquid and relaxation at the glass transition of the Zr46.75Ti8.25Cu7.5Ni10Be27.5 bulk metallic glass forming alloy, Acta Materialia, 46 (1998) 4725-4732.

[21] Z. Evenson, I. Gallino, R. Busch, The effect of cooling rates on the apparent fragility of Zr-based bulk metallic glasses, Journal of Applied Physics, 107 (2010) 123529. 